\title{
Behind the Dance: Educational, Emotional and Social Contexts in Down syndrome
}

\author{
Lays Hevércia Silveira de Farias \\ Nurse Department \\ Federal University of Sergipe \\ Lagarto, Brazil \\ lays_hevercia@hotmail.com
}

\author{
Lavinia Teixeira-Machado \\ Education in Health Department \\ Federal University of Sergipe \\ Lagarto, Brazil \\ teixeiramachado@icloud.com
}

\begin{abstract}
Down Syndrome (DS) is a genetic condition due to a change in cell division during embryonic period, characterized by some changes in neuromotor, cognitive and sensory systems, which causes some damage in daily activities. Dance has the peculiarity to integrate body and mind, promoting creativity, expression and communication, self-knowledge and self-confidence. The aim of this study was to discuss the role of dance in the socio-educational generation for people with DS. This is a descriptive, observational, interventional study. We observed dance classes and public presentations promoted benefits, especially in domains related to communication, emotion and social aspects. Dance is an educational opportunity that involves various aspects to propagate the construction of learning and it collaborates to psychomotor, social and cultural development.
\end{abstract}

Keywords: Dance1, Development2, Learning3, Down Syndrome4.

\section{INTRODUCTION}

Down Syndrome (DS) is the most common genetic condition that causes of intellectual disability (ID). DS is characterized by substantial limitations in cognitive and neuromotor functioning, and adaptative behaviour caused by the pathobiology of trisomy 21 (T21) [1][2]. Because of this, neurodevelopmental outcome is poor, besides developmental deficits in all domains (cognitive, gross motor, fine motor, receptive language, expressive language) [3].

Education is a continuous process and allows the people to be able to adapt themselves to their basic needs. Over the years, it was noticed a significant change in the learning process in general framework, but also in aspects that include groups with specific characteristics, like DS, for example [4].

The change in relevant circumstances about social inclusion fragmented thoughts about how individuals with DS should be educated. Learning is a cyclical and embracing process, it supports on equality in order to allow the basic needs to ensure independence and autonomy [5].

The prospect of inclusive education has fostered the need to understand the availability of mechanisms that can be useful for learning. Gilmore, Campbell and Cuskelly [5] and Fidler and Nadel [6] highlight collaborative education to enable learning in people with disabilities, including DS.

Dance arises from the conception of a collaborative learning, in order to incite autonomy. This education encourages the learning through body-mind action and own initiatives [7]. And dance allows the discovery of what is peculiar and proper human body [8].

Because of this, the aim of the study was to discuss the influence of dance in strength educational, emotional and social aspects, besides the sovereign autonomy in DS.

\section{METHODS}

\subsection{Design and Participants}

This descriptive, observational and interventional study approaches dance as educational, emotional and social potential for individuals with DS. The participants were excluded who refused to sign the consent form or not carried out the assessment and reassessment. 


\subsection{Protocol Procedure}

First of all, parents signed the consent form. Dance classes took place twice a week, lasting 60 minutes each, during twelve months, in a suitable classroom, at Federal University of Sergipe, Brazil.

The choreographic sequences in dance classes were aided by playful songs, they allowed know-how corporal and they encouraged communication and socialization of participants. Classes were divided in three moments: warm-up (strength and body conditioning); choreographic compositions (body coordination, memory, perception, rhythm); relaxation (final stage). In addition, choreographed pieces were prepared for public presentations.

\subsection{Assessment}

A form was used to register descriptive and anthropometric characteristics of the participants (name, age, sex, weight, height, body mass index, and relevant data to the clinical history, such as current history, past, physiological, social family histories, among others). To assess the efficacy of proposed actions in this study, it was performed through interviews related to knowledge, attitude and practice to measure knowledge, thinking and acting of the participants. The interviews provided diagnosis of achievements in the dimensions: educational (memory, skill, design and understanding of seized body movements); emotional (attitudes related to the ability to opine, predisposing, believing and having feelings to the context in evaluation); social (involves the practice itself, as well as the psychomotor, cognitive and affective domain: it is the decision-making to perform the action).

To evaluate independence, emotional, educational and social conditions, we used Functional Independence Measure (FIM): seven domains (items are scored as 1-7 (1 indicates total assistance and 7 indicates complete independence). Score ranking is 18 to 126, the lowest and highest independence, respectively [9].

The International Classification of Functioning, Disability and Health (ICF) was developed by the World Health Organization, which ranks the health and health-related aspects, besides environmental factors, including education, and social participation. The ICF defines activity as a personal task or behaviour, while participation is defined by involvement in actual situations of daily living [10].

The Mini Mental State Examination (MMSE) was used to indicate cognitive status. This test evaluates place and time orientation, memory, attention and calculation, ability to construct a sentence, language skill. The total score is 30 points, and scores less than 24 points are defined as cognitive impairment state [11][12].

\section{RESULTS AND DISCUSSION}

The study included 12 participants with DS, 4 male and 8 female, age between 13 and 30 years old, height mean $1.39(0.16) \mathrm{m}$, weight, $42.15(10.65) \mathrm{kg}$, and body mass index (BMI), 22.19(2.02) kg/m².

After dance lessons and some artistic public presentations, the participants obtained increase in FIM scores: before 65.5(7.34) and after 97.37(3.29). This increase indicated improvement of independence. Mobility, Locomotion, Communication, cognitive function and psychosocial adjustments showed noticeable improvement in the variables involving communication and social cognition, such aspects involve educational, emotional and social aspects (Table 1).

Table1. Functional Independence Measure (FIM) before and after 12 months dance classes.

\begin{tabular}{|c|c|c|c|}
\hline \multicolumn{4}{|c|}{ Functional Independence Measure (FIM) } \\
\hline & \multicolumn{3}{|l|}{$\mathrm{N}=12$} \\
\hline & Before & After & P-value \\
\hline Self Control & $3.17(0.48)$ & $3.50(0.59)$ & 0.11 \\
\hline Sphincter Control & $5(0.78)$ & $5.30(0.66)$ & 0.22 \\
\hline Mobility & $3.25(0.44)$ & $4.71(0.67)$ & $0,0001 * *$ \\
\hline Locomotion & $3.19(0.42)$ & $4.19(0.55)$ & $0,004 *$ \\
\hline Communication & $4.76(0.64)$ & $5.57(0.46)$ & $0.005 *$ \\
\hline Cognitive Function & $2.71(0.46)$ & $5.38(0.51)$ & 0.05 \\
\hline Psychosocial Adjustments & $2.55(0.66)$ & $3.11(0.42)$ & $0.004 *$ \\
\hline Total & $65.5(7.34)$ & $97.37(3.29)$ & $0.0006 * *$ \\
\hline \multicolumn{4}{|c|}{ International Classification of Functioning, Disability, and Health } \\
\hline & $84.15(4.62)$ & $39.90(5.80)$ & 0.001* \\
\hline \multicolumn{4}{|c|}{ Mini Mental State Examination (MMSE) } \\
\hline & $22.01(0.99)$ & $23.02(0.87)$ & 0.38 \\
\hline
\end{tabular}

Mean(SD). $T$ test for dependent samples. 
Dance has the peculiarity to integrate body and mind, promoting creativity, expression and communication, self-knowledge and self-confidence. Dance is an educational appliance that facilitates the development of social possibilities. Dance has a motivating effect and it works to improve the functional independence in DS.

The ICF approaches environmental aspects, such conditions influence the limitation of people with DS to leisure and work activities. The findings increased after dance classes and public presentations (Table 1). Dance promoted a deeper knowledge of the environment, including the educational, emotional and social influences, it allowed potential capability to perform functional activities and social participation of the involved population.

Dance can be able to show that it is possible to improve the life of people with DS and reduce their limitations with strategies aiming at adjusting the comprehension about their body, mind and its surroundings, considering that the environment is one of the main generators of human ability, and so it should be the focus of strategies aimed at human functionality.

That is because dance involves physical, psychological and social aspects, which are reset through leisure activities. Moreover, this art conveys communication and expression, fundamental factors in the socio-educational processes. It is multifaceted and its benefits are unrestricted, it also keeps the body active and in search of physical, mental and spiritual [8][13].

In know-how context, dance envisions a proposal that is not restricted to teaching dance steps as it is something which is expressed through cultural teachings and subjectivity. It is precisely the perception of cognitive ability of the individual who is placed as a goal.

Learning is the process by which the individual expresses the ability to deal with various situations of life after exposure to them. It determines the psychomotor development and basic neurological developments for daily living [5].

Evaluation of efficacy of dance in DS was performed through interviews related to knowledge, attitude and practice to measure comprehension, the thinking and acting of the participant in front of the actions proposed in this study to provide diagnosis of achievements in the educational, emotional and social dimensions.

Regarding the educational, emotional and social dimensions, the findings showed that participants obtained satisfactory gains: educational (remembrance, skill, conception and understanding of seized body movements); emotional (attitudes related to the ability to opine, predisposing themselves, believing and feeling part of the environment context); social (participants were assiduous and were completely involved by the practice of dance, as well as the psychomotor, affective and cognitive domains which generated decisions, actions and behaviors in society).

Although DS is characterized by substantial limitations in intellectual functioning [1][2], dance promoted adaptative behavior, due to many factors, which we could highlight the mnemonic memory, it is related to affective learning - the arts inquire in humans.

The composition of choreographies allowed the enhancement of mnemonic capacity of the participants during the lessons, and especially after exposures in front of the public. Reinders et al. [13] also noted favoring cognitive aspects, including the attention and allowed interaction with the environment, through the repetition of steps and interference the rhythm of the songs, these conditions increased the memory capacity.

That is because dance promotes cognitive development in DS, and it is notorious the influence in socio-educational process: the practice of dance not only influences the physical aspects such as improvement of the motor apparatus, but also the parameters involving cognition and emotion, essential aspects for communication and autonomy.

Dance can promote autonomy and mastery because it stimulates various skills: time and spatial adjustments, non-verbal communication, self-confidence, self-knowledge, social inclusion.

Regarding the educational process, social inclusion is constantly changing, and needs to be expanded through effective strategies. This is mostly seen in building capacity and interaction between people, and it depends on language and body-mind exploration [6].

All of these aspects contributed to the educational, emotional and social adjustments in DS through the process of dance learning. In broad suggestion, dance is essential for daily living, it causes developmental effects on human perception of its skills. In addition, dance can prepare the person to deal with such skills by strengthening self-confidence and autonomy. 


\section{Conclusion}

Besides the transformations involving the educational, emotional and social development in DS, dance showed to be essential for the evolution of the other capabilities of the human being, it is revealed as a fundamental strategy and agent facilitator of functional independence of these participants.

The established interaction between persons with DS through dance provided the inclusion of social being in search of their inherent human skills, because this art is not just a set of elaborate steps to perform an action, dance allows the comprehension, learning and performance the body as a whole, and this fact enables communication, education and interaction between DS and all the kinds of people in society.

Dance assembled physical, psychological and social aspects, mainly it provided autonomy and freedom of expression in the sense that "I know who I am, I know I can and I can be-do part of the world".

\section{REFERENCES}

[1] X.L. D'Ardhuy, J.O. Edgin, C. Bouis, S. de Sola, C. Goeldner, P. Kishnani, et al. Assessment of cognitive scales to examine memory, executive function and language in individuals with Down Syndrome: implications of a 6-month observational study. Front Behav Neurosci, 9:300, 2015.

[2] D. Nizetic, C.L. Chen, W. Hong, E.H. Koo. Inter-dependent mechanisms behind cognitive dysfunction, vascular biology and Alzheimer's dementia in Down Syndrome: multi-faceted roles of APP. Front Behav Neurosci, 1; 9: 299, 2015.

[3] S. Tapp, T. Anderson, J. Visootsak. Neurodevelopmental outcomes in children with Down syndrome and infantile spasms. J Pediatr Neurol. 2015 Jun;13(2):74-77.

[4] S. Buckley, G. Bird, B. Sacks, T. Archer. A comparison of mainstream and special education for teenagers with Down syndrome: implications for parents and teachers. Downs Syndr Res Pract, 9(3): 54-67, 2006.

[5] L. Gilmore, J. Campbell, M. Cuskelly. Developmental expectations, personality stereotypes, and attitudes towards Inclusive Education: community and teacher views of Down syndrome. Intern $\mathrm{J}$ of Disability, Development and Education, 50(1): 65-76, 2003.

[6] D.J. Fidler, L. Nadel. Education and children with Down syndrome: Neuroscience, development, and intervention. Mental Retardation and Developmental Disabilities Research Reviews, 13(3): 262-71, 2007.

[7] L. Teixeira-Machado, J. DeSantana. Dance therapy and quality of life in individuals with physical deficit: randomized controlled trial. Brazilian Journal of Quality of Life, 5(1):39-52, 2013.

[8] L. Teixeira-Machado, J. DeSantana. Dance therapy improves quality of life in individuals with neuromotor disorders: randomized controlled trial. International Journal of Humanities Social Sciences and Education, 2(4):84-92, 2015.

[9] J.M. Linacre, A.W. Heinemann, B.D. Wright, C.V. Granger, B.B. Hamilton. The structure and stability of the Functional Independence Measure. Arch Phys Med Rehabil, 75(2):127-32, 1994.

[10] WHO: International classification of functioning, disability and health, 2001. http://www.who.int/classifications/icf/en/.

[11] M.F. Folstein, S.E. Folstein, P. McHugh. Mini-Mental State: a practical guide for grading the cognitive state of patients for the clinician. Journal of Psychiatric Research, 12:189-98, 1975.

[12] B. Punchik, A. Shapovalov, T. Dwolatzky, Y. Press. Visual-spatial perception: a comparison between instruments frequently used in the primary care setting and a computerized cognitive assessment battery. Clin Interv Aging, 10:1881-7, 2015.

[13] N. Reinders, P.J. Bryden, P.C. Fletcher. Dancing with Down syndrome: a phenomenological case study. Research in Dance Education, 16(3):291-307, 2015. 


\section{AUTHOR'S BIOGRAPHY}

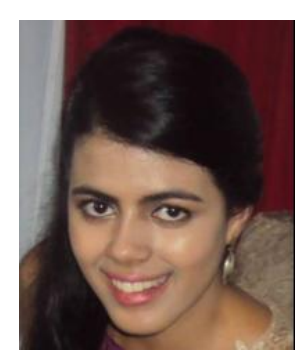

Lays Hevércia Silveira de Farias, is studying Nursing at Federal University of Sergipe. She is scholarship holder of extension project: TALT: dance and music as tools to support social inclusion.

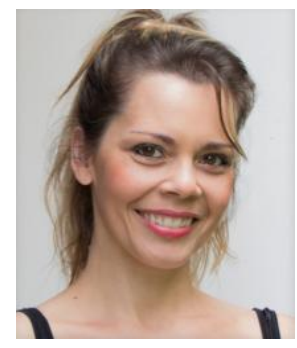

Lavinia Teixeira-Machado, $\mathrm{PhD}$ in Health Sciences, expert in biomechanics and Kinesiotherapy Functional, degree in Physical Therapy, broadcaster, professional dancer. Professor of Health in Education Department at Federal University of Sergipe, Brazil. She is a researcher and collaborator in the following research groups: Pain and Mobility; Art, Diversity and Contemporary. Member of Brazilian Association of Neurofunctional Physical therapy (ABRAFIN), and the American Dance Therapy Association (ADTA). Experience in neurofunctional and dance education, acting on the following topics: body awareness, classical and contemporary dance, physical therapy and people with disabilities. 